\title{
Pathophysiologic Aspects of Hypertension
}

\begin{abstract}
Hypertension is defined as a sustained increase in blood pressure. Historically, the level of blood pressure designating hypertension has been a systolic blood pressure of $140 \mathrm{~mm} \mathrm{Hg}$ or more and/or a diastolic blood pressure of $90 \mathrm{~mm} \mathrm{Hg}$ or more. However, the risk imparted with increasing blood pressure is continuous, such that the level of blood pressure must be considered within the context of the overall cardiovascular risk profile. Clearly, antihypertensive treatment helps to prevent both cardiovascular and cerebrovascular events, the dreaded complications of increased blood pressure. Nevertheless, hypertension remains unrecognized or inadequately treated in the great majority of patients. This work aims to review the essential key element for the understanding of hypertension's pathophysiology.
\end{abstract}

Keywords: : Pathophysiology; Blood Pressure and Hypertension.

\section{*Corresponding Author:}

Mohamed Eddouks,

Faculty of Sciences and Techniques Errachidia. Moulay Ismail University, BP 21, Errachidia, Morocco.

Tel: 00212535574497

Fax: 00212535574485

E-mail: mohamed.eddouks@laposte.net

Recieved: January 02, 2015

Accepted: February 20, 2015

Published: February 24, 2015

Citation: Eddouks M*, Hebi M, EL Bouhali B, Zeggwagh N (2015) Pathophysiologic Aspects of Hypertension. Int J Diabetol Vasc Dis Res, 3(1) 76-82. doi: http://dx.doi.org/10.19070/2328-353X-1500015

Copyright: Eddouks $\mathbf{M}^{\circ} 2015$. This is an open-access article distributed under the terms of the Creative Commons Attribution License, which permits unrestricted use, distribution and reproduction in any medium, provided the original author and source are credited.

\section{Introduction}

High blood pressure (hypertension) is one of the most common chronic medical conditions in the developed world and is rapidly becoming a major problem in developing countries. It is estimated that about $20 \%$ of the world's adult population, or some 690 million adults, suffer from hypertension [1-3]. Blood pressure in the population is normally distributed in a bell-shaped curve with a slight skew towards higher readings (Figure 1). Thus, there is no upper limit of 'normal' blood pressure beyond which cardiovascular complications occur. Rather, blood pressure levels, both systolic and diastolic, have been shown to be positively and continuously associated with the risk of stroke, coronary heart disease and other forms of target organ damage. However, in order to facilitate patient assessment and management, an arbitrary definition of hypertension has been established $[4,5]$.

Both the Joint National Committee on Prevention, Detection,
Evaluation and Treatment of High Blood Pressure (JNC) in the United States of America and the World Health OrganizationInternational Society of Hypertension (WHO-ISH) now define hypertension in adults as a systolic blood pressure (SBP) of 140 $\mathrm{mm} \mathrm{Hg}$ or more or a diastolic blood pressure (DBP) of $90 \mathrm{~mm}$ $\mathrm{Hg}$ or more on at least two subsequent occasions. Isolated systolic hypertension is defined as a SBP $140 \mathrm{~mm} \mathrm{Hg}$ and a DBP $90 \mathrm{~mm}$ $\mathrm{Hg}$. The great majority, at least $95 \%$ of cases of hypertension, can be classified as primary or essential hypertension because no secondary causes such as neurovascular disease, renal failure, phaeochromocytoma or hyper-aldosteronism are present [6-9].

\section{Regulation of Arterial Blood Pressure}

Although the cardiovascular system is capable of maintaining blood flow and cardiac function in the absence of any nervous system input, addition of neural control mechanisms allows very precise, short-term (second-to-second and minute-to-minute) cardiovascular regulation. Studies over the last 30 years demonstrate that the nervous system can also contribute to long-term cardiovascular and blood pressure (BP) regulation. In several animal models and in subsets of human hypertensive patients, chronic activation of the nervous system appears to contribute to persistent hypertension and the resulting target organ damage [10-13].

The final common pathway for the contribution of the nervous system to chronic arterial pressure control involves the sympathetic and parasympathetic divisions of the autonomic nervous and the associated neurohormonal systems primarily regulated by the hypothalamus. Most experimental evidence suggests the parasympathetic nervous system is much less involved in BP regulation and hypertension than the sympathetic nervous and neurohormonal systems [14-17]. The autonomous nervous system contributes to the development and maintenance of hypertension through stimulation of cardiac output in heart, fluid retention in kidney and increased vascular resistance in peripheral vasculature. 
Figure 1. Frequency distribution of diastolic blood pressure of 158906 persons aged 30-69 years in the Hypertension Detection and Follow-Up Program.

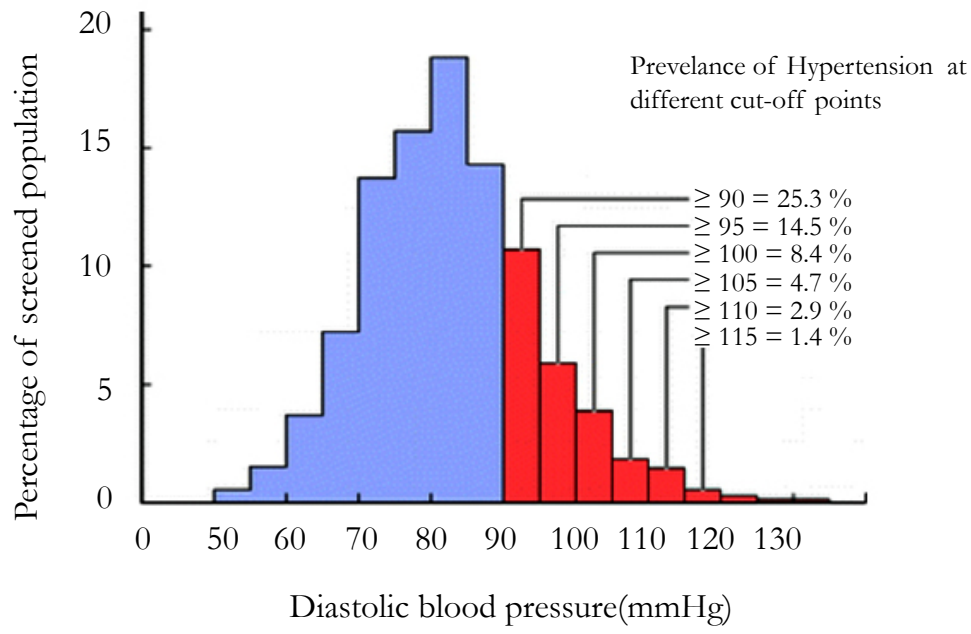

Both the sympathetic and the parasympathetic nervous systems are known to innervate blood vessel walls and consequently regulate contraction and wall tension. Elevated sympathetic activity induces an increase in blood pressure, by causing peripheral vasoconstriction, reducing venous capacitance, and affecting renal sodium and water excretion. For example, in response to increased sodium intake, sodium-sensitive receptors, present in the circumventricular organs and hypothalamic paraventricular nucleus, inhibit renal sympathetic nerve activity to facilitate natriuresis, thus maintaining fluid and electrolyte homeostasis and normotension.

\section{Peripheral Autonomic Nervous System}

Efferent Nerves: The autonomic nervous system includes sympathetic and parasympathetic divisions and the associated afferent (sensory) feedback nerves that affect each division. Sympathetic and parasympathetic motor neuron cell bodies are found in peripheral ganglia [18-21].

In the sympathetic nervous system (SNS), cell bodies of neurons lie in ganglia that are immediately lateral to the spinal cord (paravertebral) or anterior to the vertebral column (prevertebral). The prevertebral neurons primarily innervate visceral organs, including the heart and kidney, whereas the paravertebral neurons project more prominently to blood vessels throughout the body. Irrespective of their location, all sympathetic ganglia neurons synapse with pre-ganglionic neurons that lie in the thoracic portion of the spinal cord [22-24].

Parasympathetic motor neuronal cell bodies are located in ganglia that are very close to the organ that is innervated. These ganglion cells are innervated by neuronal cell bodies that are in the medulla (for organs above the transverse colon) or the sacral spinal cord (for organs below the transverse colon) [18, 19, 25].

Afferent Nerves: Sensory afferent feedback from the innervated tissue is projected back through the ganglia to the central nervous system (CNS). Most sympathetic afferents terminate in the spinal cord at the level that correlates with the position of the preganglionic cell bodies (e.g., the lower thoracic spinal cord is the usual location of renal sympathetic pre-ganglionic neurons and provides most of the renal alpha sympathetic sensory feedback to the CNS). Parasympathetic sensory innervations follows the projection pattern of the motor fibers, and most of it terminates in the dorsal brain stem $[18,21,26-28]$.

\section{Cardiovascular Monitoring Systems}

Arterial baro-receptors are stretch-sensitive sensory nerve endings located in the carotid sinuses and aortic arch that function as arterial pressure sensors (Figure 2). Afferent (sensory) baroreceptor activity is transmitted to the nucleus tractus solitarii in the medulla oblongata, where the signals are integrated and relayed through a network of central neurons that determine efferent autonomic outflow $[14,16,22,29,30]$.

Baroreceptors: The brain continuously monitors arterial pressure through stretch receptors (mechanoreceptors) attached to vagal and glossopharyngeal axons innervating the aortic arch and carotid bifurcation (aorto-carotid or high-pressure baroreceptors). In parallel, blood volume is monitored by branches of the vagus nerve innervating the cardiac atria and ventricles (cardiopulmonary or low-pressure baroreceptors). Baroreceptors located elsewhere in the body (e.g., the kidney) serve a similar function. Although baroreflex abnormalities do not appear to lead directly to hypertension, the loss of these reflexes greatly increases BP lability and thereby accelerates end-organ damage [18, 19, 26, 30].

Chemoreceptors: Chemoreceptor's sensitive to vascular $\mathrm{O}_{2}$ deficiency, $\mathrm{CO}_{2}$ excess, and $\mathrm{H}^{+}$excess are found in the carotid bodies and adjacent to the aorta. These receptors are not as important to arterial pressure regulation as are the mechanoreceptors under usual conditions but appear to play a role in arterial pressure regulation during extreme conditions such as hypoxia $[21,28,30,31]$.

Osmoreceptors: Osmoreceptors found in several areas of the brain and in the periphery can also modify arterial pressure; recent studies have highlighted the importance of hepatic osmoreceptors in cardiovascular regulation $[15,17,26,32]$.

\section{Local Modulation of Neurotransmission}

Less conventional forms of synaptic transmission may be important to the role of the SNS in arterial pressure regulation. It has 
Figure 2. Cardiopulmonary and arterial baroreflex neural pathways involved in cardiovascular homeostasis and blood pressure (BP) regulation. Locations of arterial baroreceptors are indicated by filled circles.

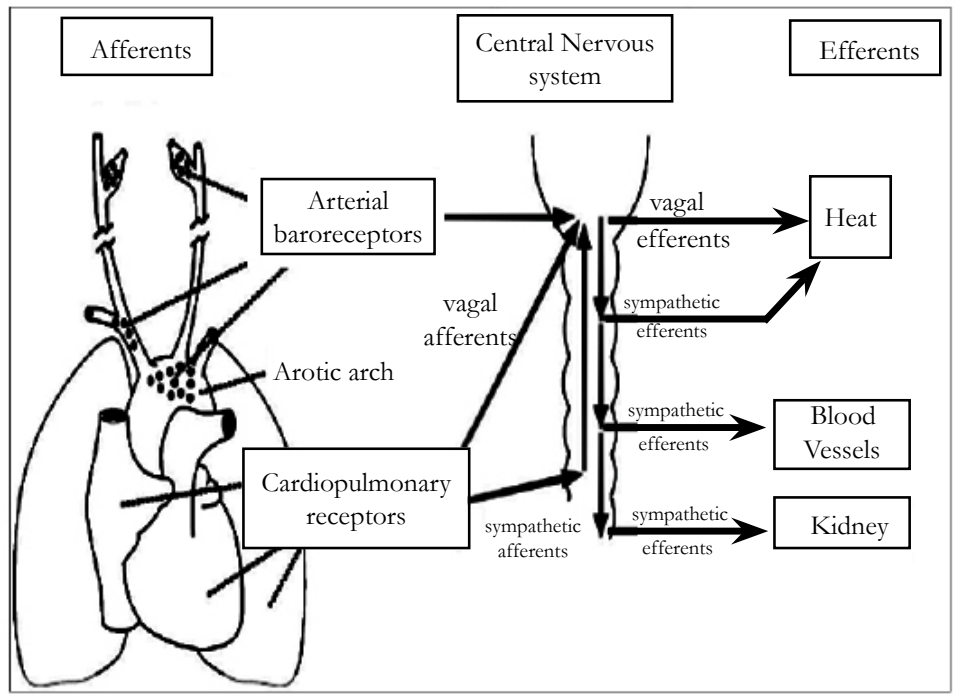

been demonstrated that neurotransmitters released from efferent (motor) nerve terminals in the kidney can alter the ability of afferent (sensory) axons to send information to the CNS [19, 21]. In addition, some studies have shown that peripheral afferent nerves directly innervate neurons in the sympathetic ganglia and give rise to sensory feedback control that does not go through the CNS $[25,32,33]$.

\section{Neurohormones}

Other neurotransmitters and neuromodulators released by sensory neurons have profound effects on the target organs. Perhaps the best example is calcitonin gene related peptide (CGRP). Release of CGRP from peripheral afferent neurons onto the blood vessels, is a potent cause of vasodilation. Recent studies in the rat suggest that the release of CGRP is inhibited by $\alpha 2$ adrenoreceptor activation. Therefore, the overabundance of norepinephrine in a target tissue could engender vasoconstriction not only directly by stimulation of $\alpha 1$-adrenoceptors, but also indirectly through inhibition of CGRP release $[14,16,17,34]$.

\section{Renal Sympathetic Nerves and Extracellular Fluid Volume Regulation}

Renal Sympathetic Innervations: The kidney is innervated only by the sympathetic nervous system with postganglionic fibers arising from spinal segments, with great variability among individuals. Sympathetic nerves are characterized by strings of varicosities along their axons that contain the neurotransmitter, norepinephrine. Renal sympathetic nerve fibers track mainly through the cortex of the kidney, passing in close proximity to renal resistance vessels (afferent and efferent arterioles), and nephrons, especially proximal convoluted tubules and thick ascending limbs of the loops of Henley [32].

Functions of Renal Sympathetic Nerves: Low-level activation of the renal nerves has little influence on renal hemodynamics, acting instead to increase renin secretion and tubular sodium and water reabsorption. High rates of renal nerve activity cause shortterm reductions in both renal blood flow and glomerular filtra- tion but have little influence on fluid balance. In contrast, a small rise in renal nerve activity for extended periods can have a major impact on extracellular fluid volume (ECFV). The actions of the renal sympathetic nerves on these various functions are summarized in Figure 3 [27, 28, 31].

Tubular Sodium Reabsorption: Tubular epithelial cells are stimulated by norepinephrine released at neuro-effector junctions, with an ensuing activation of alpha-adrenoceptors on basolateral membranes. This causes an increase in sodium $\left(\mathrm{Na}^{+}\right)$hydrogen $\left(\mathrm{H}^{+}\right)$exchanger activity at the apical membrane, allowing sodium to enter the cell, whereupon it is pumped out of the cell through $\mathrm{Na}^{+} / \mathrm{K}^{+}$adenosine triphosphatase (ATPase) located in basolateral membranes. Water of hydration follows $\mathrm{Na}^{+}$ions through (transcellular route) and between (paracellular route) these high permeability epithelial cells $[20,21]$.

Renin-Angiotensin-Aldosterone System Stimulation: Neural-mediated release of renin occurs when norepinephrine stimulates $\alpha 1$-adrenoceptors on the juxta glomerular (granular) cells of the afferent arterioles located at the entrance to the glomerulus. The subsequent generation of angiotensin II (Ang II) has both intra- and extra-renal actions that affect renal $\mathrm{Na}+$ and fluid handling as mediated by AT1 receptor stimulation. Within the kidney, Ang II constricts afferent and efferent arterioles; constriction of the efferent arterioles is especially important in maintaining glomerular filtration pressure in low flow and/or hypotensive states. Ang II also acts rapidly and directly on proximal epithelial cells to increase fluid reabsorption. Aldosterone release prompted by Ang II, in addition, results in distal nephron and collecting duct $\mathrm{Na}^{+}$ reabsorption. Together, these neural effects regulate fluid volume homeostasis in such a way that both rapid and/or chronic adaptive responses are possible [14-16].

Sensory information from many body systems is integrated in the hypothalamus and brainstem guiding autonomic regulation of sympathetic and parasympathetic nerves (Figure 4).

Afferent fibers stimulated by mechanoreceptors and chemoreceptor's in the somatic (muscle and skin) and visceral (gut, liver, and 
Figure 3. The innervations of the afferent and efferent resistance arterioles, renin-containing (juxta glomerular) cells, epithelial cells of the kidney, and the norepinephrine (NE) stimulated adrenoceptors involved.



kidneys) systems provide input to the CNS and generally stimulate efferent sympathetic nerves. Higher cortical neurons also provide input to cardiovascular control centers by way of psychological stressors and environmental conditions. The most important regulators of efferent renal sympathetic nerve activity are the high pressure (aortocarotid) and low-pressure (cardiopulmonary) baroreceptors [35-37].

\section{Pathophysiology of Hypertension}

Hypertension is a haemodynamic disorder in which increased arterial pressure may be associated with an increased cardiac output or increased total peripheral resistance. In most patients it is an increased total peripheral resistance that produces the increased arterial pressure. A large number of pathophysiological factors have been implicated in the genesis of hypertension, including: (1) increased sympathetic nervous system activity, possibly related to increased responsiveness to psychosocial stress; (2) overproduction of sodium-retaining hormones and vasoconstrictors such as endothelin or thromboxane; (3) increased sodium intake; (4) inappropriate secretion of renin; and (5) decreased production of vasodilating substances such as nitric oxide or prostaglandins $[2,5,38,40]$.

Most cases of hypertension result from the interplay of genetic and environmental factors, with $25-40 \%$ of blood pressure variation being genetically determined. High blood pressure is a complex trait that does not follow classical Mendelian rules of inheritance attributable to a single gene locus, except for several rare forms of monogenic hypertension. Rather, it is a polygenic and multifactorial disorder in which the interaction of several genes and environmental factors such as alcohol intake, physical exercise, diet (calories, micronutrients) and stress is important [37, 41, 43].

Hypertension in isolation leads to initial and medial thickening of blood vessels, a condition that has been referred to as nodular arteriosclerosis. These lesions may result in fibrous plaque and thrombus formation, which is probably responsible for ischemia and infarction of the brain and kidneys in patients with severe chronic hypertension. More commonly, hypertension coexists with hyperlipidemia, and the combination of risk factors leads to the formation of lipid-rich atherosclerotic plaques, eventually resulting in coronary and cerebrovascular events [9, 44]. Aging, Hypertension, and Arterial Function

Blood pressure and arterial stiffness: Elevated BP can increase arterial stiffness (and elastic modulus) by functional and structural mechanisms. With an increase in luminal pressure, the loadbearing elastic lamellae stretch and become stiffer and the loosely woven collagen web is progressively engaged. Since collagen is several orders of magnitude stiffer than elastin, transfer of load from elastin to collagen is associated with a marked nonlinear increase in functional stiffness (and elastic modulus) of the arterial wall. Contraction of vascular smooth muscle also tends to favor increased stiffness $[11,12,45]$.

Wall Composition: Central arterial elasticity is critically dependent on normal content and function of the matrix protein elastin, whose half-life of 40 years is one of the longest in the body. Despite this stability, fatigue of elastin fibers and lamellae from the accumulated cyclic stress of more than 2 billion aortic expansions often has occurred by the sixth decade of life. Eventual fracturing and disarray of elastin is accompanied by structural changes of the extracellular matrix that include proliferation of collagen and deposition of calcium. Humoral factors, cytokines, and oxidative metabolites may also play a pathogenic role. This pathological process, classically termed as arteriosclerosis, results in increased stiffness of the aortic wall at any ambient pressure [29, 46, 47$]$.

\section{Disorders of Extracellular Fluid Volume Regulation}

Several pathophysiological states are associated with disordered ECFV regulation and raised systemic and renal sympathetic outflow, including hypertension, heart failure, and cirrhosis. In hypertension, the increased sympathetic drive may originate from the central nervous system itself, either through higher cortical input pathways and/or from other sources of dysregulated sensory input $[8,21]$. Heart failure is a state of systemic underperfusion, where decreasing cardiac output progressively fails to meet the metabolic needs of the body $[34,35]$. A major systemic response to this hypoperfusion state is reflex sympatho-excitation, presumably intended to drive the heart and restore output. In cirrhosis, raised venous pressure and reduction in functioning liver mass activates hepatic receptors and in so doing engenders reflex sympatho-excitation [38]. 
Figure 4. Sensory systems providing input to the central nervous system and the pathways of sympathetic nerve-mediated renal functional responses.

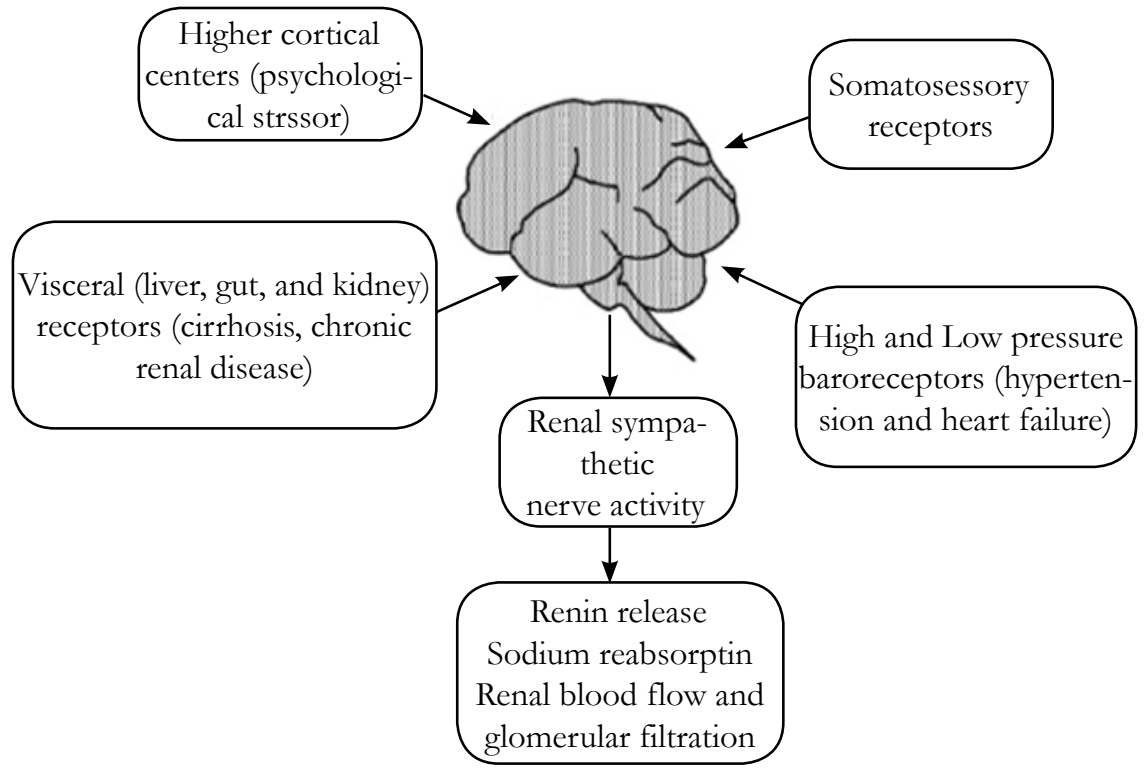

An important renal consequence in all these states is neurallydependent $\mathrm{Na}^{+}$retention leading to an increased ECFV [13]. In hypertension, this contributes to the persistence of the hypertensive state. In heart failure, any rise in ECFV generally increases preload, which both compromises the function of the failing heart and contributes to congestive symptomatology. As cirrhosis evolves and volume expansion occurs there is also a concomitant decrease in plasma proteins and osmotic pressure, factors which together exacerbate the edema state [25].

There are two primary therapeutic strategies in these pathophysiological conditions: (1) reducing sympathetic drive to the kidney and hence any neurally-induced $\mathrm{Na}^{+}$retention and (2) use of diuretics and vasoactive drugs that affect renal tubular re-absorptive processes in such a manner that excess $\mathrm{Na}^{+}$and water is mobilized.

\section{Obesity-Related Hypertension}

Epidemiologic studies have shown that the prevalence of obesity in children, adolescents, and adults is increasing worldwide. In the Framingham Heart Study, excess weight was associated with hypertension in $78 \%$ of men and $65 \%$ of women. Other investigators have found that central (truncal or visceral) obesity, rather than peripheral adiposity, is closely associated with hypertension. The cause of hypertension in obesity is complex and multifactorial, including hemodynamic, metabolic, and endocrine mechanisms [13].

Mechanisms Of Obesity-Related Hypertension: Central obesity is the most common condition associated with insulin resistance and the consequent hyperinsulinemia. The resistance to insulin, however, is selective and not uniform in all tissues. In animal experiments insulin increases absorption of sodium in the diluting segment of the distal nephron, with a manifest effect of salt and water retention; it also increases adrenergic activity and causes vascular smooth-muscle hypertrophy. Insulin resistance and hyperinsulinemia may also impair the insulin-mediated vascular signaling pathways associated with vasorelaxation, mechanisms that may link hyperinsulinemia and insulin resistance to the development of hypertension [23, 25].
Most of the insulin-resistance hypertension connection has been demonstrated in animal experiments. Acute and chronic studies that induce hyperinsulinemia in humans have failed to achieve consistent effects on blood pressure (BP) or other BP-raising mechanisms such as sodium reabsorption or sympathetic activity (SA). Consequently, the association between hyperinsulinemia and hypertension remains somewhat controversial. Some investigators believe that insulin resistance and hyperinsulinemia may only minimally contribute to the relationship between obesity and hypertension $[22,42]$.

Sympathetic Nervous System: Peripheral catecholamine levels or SA are not always elevated in obese compared to non-obese subjects; however, regional organ-specific SA in muscle and kidneys is elevated. The regionally elevated SA in obese subjects may, in part, explain the increased incidence of hypertension, arrhythmias, and angina pectoris that characterize obesity-hypertension $[15,28]$.

Dogs made obese by overfeeding demonstrate activated renal sympathetic nerve traffic and increased BP. Renal denervation in these animals attenuates sodium retention and prevents the development of hypertension. Increased SA in the kidneys of obese patients has been found in several studies, and hyperleptinemia appears to be the most important mechanism that triggers the increase in SA in these subjects [6].

\section{Vascular and Renal Nitric Oxide and hypertension}

Nitric oxide (NO) is an endogenously produced, freely diffusible gas with a half-life of several seconds. NO functions as an endogenous intracellular and intercellular messenger that is involved in many pathophysiological responses, especially regional blood flow regulation and sodium $\left(\mathrm{Na}^{+}\right)$per water excretion. Cardiovascular and renal health also depends on the pleiotropic effects of NO [9, 22].

Studies in humans indicate that essential hypertension may be associated with a decrease in nitric oxide generation. Experiments in animal models, however, suggest that production of nitric oxide by these animals may be reduced, unimpaired or even increased, depending on the model of hypertension. Thus, it has been hy- 
Figure 5. Pathogenesis of obesity-related hypertension. HDL, high-density lipoprotein; SNS, sympathetic nervous system; ANP, atrial natriuretic peptide; RAAS, renin-angiotensin-aldosterone system; CKD, chronic kidney disease; CVD, cardiovascular disease; T2DM, type 2 diabetes.



pothesized that in relation to nitric oxide there may be two forms of hypertension [7, 28]. In one type, increased vasoconstrictor activity (which may be caused by different factors) leads to an increase in nitric oxide generation as a compensatory mechanism; in this situation there may be normal or increased sodium excretion since nitric oxide plays a role in facilitating sodium handling. The other type may depend on a deficiency in nitric oxide generation in the vessel wall, which would be accompanied by impaired renal sodium handling. Thus, the first form of hypertension would be associated with abnormally high vasoconstrictor activity, whereas in the second form normal levels of vasoconstrictors will in effect behave as excessive owing to the lack of counteracting nitric oxide-dependent vasodilator tone $[22,33]$.

\section{Emerging Aspects Of Hypertension}

Recently, it has been suggested that the inflammation in the brainstem may underlie neurogenic hemodynamic disorder [48]. Substances with a potential anti-inflammatory, antioxidant and endothelium-protecting action in the central nervous system, such as melatonin, might become important players in the therapeutic targeting. The antihypertensive effect of melatonin was demonstrated in experimental and clinical hypertension. This neurohormon has been shown to lower inflammation and free radical burden, correct endothelial dysfunction, to protect organs and to shift the balance between the sympathetic and parasympathetic system in favor of the parasympathetic system. In addition, sodium/water excretion, adrenal steroids and protein-derived peptides are other factors controlling hypertension [48]. There are many efforts targeting the understanding of the pathophysiologic mechanisms of hypertension and many new strategies are being investigated in order to manage hypertension. In this view, modulation epigenetic regulation of genes involved in BP homeostasis, device based interventions, including baroreceptor activation and renal denervation therapy are some emerging therapies for hypertension [49].

\section{Conclusion}

High blood pressure is a major health concern worldwide and will continue to grow in importance as the population ages and developing nations become more urban and industrialized. It is imperative to enhance both clinician and public awareness of the consequences and of the readily available treatments for hypertension. Also, major education campaigns promoting healthy lifestyles need to be initiated, as these may aid in the primary prevention of hypertension.

\section{Grants}

This work has been supported by the "MESRSFC: Appel à projets dans les domaines prioritaires de la recherche scientifique et du développement technologique".

\section{References}

[1]. Epstein BJ (2010) Aliskiren and valsartan combination therapy for the management of hypertension. Vasc Health Risk Manag; 6: 711-722.

[2]. Ferrario CM, Smith RD (2010) Role of olmesartan in combination therapy in blood pressure control and vascular function. Vasc Health Risk Manag; 6: 701-709.

[3]. McIntyre RS, Park KY, Law CW, Sultan F, Adams A, et al.( 2010) The association between conventional antidepressants and the metabolic syndrome: a review of the evidence and clinical implications. CNS Drugs; 24: 741-753.

[4]. Gidullin NSH, Valeeva KF, Gassanov N, Zagidullin SHZ (2010) Value of endothelial dysfunction in cardiovascular diseases and methods of its correction with drugs. Kardiologiia; 50: 54-60.

[5]. Dejana E (2010) The role of wnt signaling in physiological and pathological angiogenesis. Circ Res; 107: 943-952.

[6]. Ulualp SO (2010) Snoring and obstructive sleep apnea. Med Clin North Am; 94: 1047-1055.

[7]. Olteanu D, Dlupu (2010) The kidney in cirrhosis with portal hypertension. J Med Life; 3: $175-177$

[8]. Alan B, Nalbantgil S (2010) Genetic, cellular and molecular mechanisms of pulmonary arterial hypertension. Anadolu Kardiyol Derg; 10: 9-13.

[9]. Nguyen A, Thorin-Trescases N, Thorin E (2010) Working under pressure: coronary arteries and the endothelin system. Am J Physiol Regul Integr Comp Physiol; 298: R1188-R1194.

[10]. Brooks VL, Dampney RA, Heesch CM (2010) Pregnancy and the endocrine regulation of the baroreceptor reflex. Am J Physiol Regul Integr Comp Physiol; 299: R439-R451.

[11]. Hill MA, Yang Y, Ella SR, Davis MJ, Braun AP (2010) Large conductance, $\mathrm{Ca}^{2+}$-activated $\mathrm{K}+$ channels $(\mathrm{BKCa})$ and arteriolar myogenic signaling. FEBS 
Lett; 584: 2033-2042.

[12]. Yokoyama U, Minamisawa S, Ishikawa Y (2010) Regulation of vascular tone and remodeling of the ductus arteriosus. J Smooth Muscle Res; 46: 77-87.

[13]. Fedorova OV, Shapiro JI, Bagrov AY (2010) Endogenous cardiotonic steroids and salt-sensitive hypertension. Biochim Biophys Acta ; 1802: 12301236

[14]. Kougias P, Weakley SM, Yao Q, Lin PH, Chen C (2010) Arterial baroreceptors in the management of systemic hypertension. Med Sci Monit; 16: RA1-RA8.

[15]. Rossi R, Nuzzo A, Modena MG (2009) The management of pre-hypertension and metabolic syndrome. Minerva Cardioangiol 57: 723-731.

[16]. Amann M, Kayser B (2009) Nervous system function during exercise in hypoxia. High Alt Med Biol; 10: 149-164.

[17]. Ogoh S, Ainslie PN (2009) Cerebral blood flow during exercise: mechanisms of regulation. J Appl Physiol; 107: 1370-1380.

[18]. Ainslie PN, Ogoh S (2010) Regulation of cerebral blood flow in mammals during chronic hypoxia: a matter of balance. Exp Physiol; 95: 251-262.

[19]. Floras JS (2009) Sympathetic nervous system activation in human heart failure: clinical implications of an updated model. J Am Coll Cardiol; 54: 375-385.

[20]. Prakash ES. Fink GD (2010) Could hypertension possibly be adaptive? Clin Exp Pharmacol Physiol; 37: e99-e106.

[21]. Aoyagi T, Koshimizu TA, Tanoue A (2009) Vasopressin regulation of blood pressure and volume: findings from V1a receptor-deficient mice. Kidney Int; 76: 1035-1039.

[22]. Valdes G, Kaufmann P, Corthorn J, Erices R, Brosnihan KB, et al. (2009) Vasodilator factors in the systemic and local adaptations to pregnancy. Reprod Biol Endocrinol ; 7: 79.

[23]. Cravo SL, Campos RR, Colombari E, Sato MA, Bergamaschi CM, et al. (2009) Role of the medulla oblongata in normal and high arterial blood pressure regulation: the contribution of Escola Paulista de Medicina UNIFESP. An Acad Bras Cienc; 81: 589-603.

[24]. Califano JP, Reinhart-King CA (2010) Exogenous and endogenous force regulation of endothelial cell behavior. J Biomech; 43: 79-86.

[25]. Dorrington KL, Pandit JJ (2009) The obligatory role of the kidney in longterm arterial blood pressure control: extending Guyton's model of the circulation. Anaesthesia; 64: 1218-1228.

[26]. Napoli C, Ignarro LJ (2009) Nitric oxide and pathogenic mechanisms involved in the development of vascular diseases. Arch Pharm Res; 32: 11031108

[27]. Postnov IUV (2009) Energy-dependent pathogenesis in chronic hypertension. Arkh Patol; 71: 3-11.

[28]. Scheuer DA (2010) Regulation of the stress response in rats by central actions of glucocorticoids, Exp Physiol; 95: 26-31.

[29]. Caprioli J, Coleman AL (2010) Blood Flow in Glaucoma Discussion, Blood pressure, perfusion pressure, and glaucoma. Am J Ophthalmol; 149: 704712
[30]. Joyner MJ, Casey DP (2009) The catecholamines strike back. What NO does not do. Circ J ; 73: 1783-1792.

[31]. Dumitrescu D, Erdmann E, Rosenkranz S (2010) PDE5 inhibitors in the treatment of pulmonary hypertension. Pharm Unserer Zeit; 39: 391-396.

[32]. Mattson DL (2009) Measuring kidney function in conscious mice. Methods Mol Biol; 573: 75-94.

[33]. Sigmund CD (2010) Endothelial and vascular muscle PPARgamma in arterial pressure regulation: lessons from genetic interference and deficiency. Hypertension; 55: 437-444.

[34]. Cordina SM , Qureshi (2009) Syncope and carotid artery stenosis. Minerva Med; 100: 321-327.

[35]. Chhiber SS, Singh JP (2010) Acute spontaneous subdural hematoma of arterial origin: a report of four cases and review of literature. Neurol India; 58 : 654-658.

[36]. Gaspar L, Poliak P, Makovn'k M, Hlinst'kov S, Krahulec B, et al. (2010) Obesity and arterial hypertension. Vnitr Lek; 56: 1074-1077.

[37]. Kayikioğlu M (2010) The etiopathogenesis of pulmonary hypertension: inflammation, vascular remodeling. Anadolu Kardiyol Derg; 10: 5-8.

[38]. Bankir L, Bichet DG, Bouby N (2010) Vasopressin V2 receptors, ENaC, and sodium reabsorption: a risk factor for hypertension?. Am J Physiol Renal Physiol; 299: F917-F928.

[39]. Voulgari C, Papadogiannis D, Tentolouris N (2010) Diabetic cardiomyopathy: from the pathophysiology of the cardiac myocytes to current diagnosis and management strategies. Vasc Health Risk Manag; 6: 883-903.

[40]. Lee HY, Oh BH (2010) Aging and arterial stiffness. Circ J; 74: 2257-2262.

[41]. Taglieri N, Koenig W, Kaski JC (2010) Cystatin C and cardiovascular risk. Ann Biol Clin (Paris); 68: 517-529.

[42]. Yuda S, Shimamoto K, Watanabe N (2010) Clinical applications of strain rate imaging for evaluation of left atrial function. Rinsho Byori; 58: 799808.

[43]. Kapur VK (2010) Obstructive sleep apnea: diagnosis, epidemiology, and economics. Respir Care; 55: 1155-1167.

[44]. Greene SA (2010) Anesthesia for patients with neurologic disease. Top Companion Anim Med ; 25: 83-86.

[45]. Kšhler R, Ruth P (2010) Endothelial dysfunction and blood pressure alterations in K+-channel transgenic mice. Pflugers Arch; 459: 969-976.

[46]. Zanutto BS, Valentinuzzi ME, Segura ET (2010) Neural set point for the control of arterial pressure: role of the nucleus tractus solitarius. Biomed Eng Online; 9: 4.

[47]. Alper AB, Calhoun DA, Oparil S (2001) Hyprenstion. Wiley. DOI: 10.1038/npg.els.0002303.

[48]. Kaustav Majumder, Jianping Wu (2015) Molecular Targets of Antihypertensive Peptides: Understanding the Mechanisms of Action Based on the Pathophysiology of Hypertension. Int. J. Mol. Sci; 16, 256-283.

[49]. Eric K. Judd1, Suzanne Oparil (2013) Novel strategies for treatment of resistant hypertension. Kidney International Supplements; 3: 357-363. 